Meta

Journal des tradlucteurs

Translators' Journal

\title{
MÉTÉO : un système à l'épreuve du temps
}

John Chandioux et Marie-France Guéraud

Volume 26, numéro 1, mars 1981

L'informatique au service de la traduction

Machine Aids to Translation

URI : https://id.erudit.org/iderudit/002213ar

DOI : https://doi.org/10.7202/002213ar

Aller au sommaire du numéro

Éditeur(s)

Les Presses de l'Université de Montréal

ISSN

0026-0452 (imprimé)

1492-1421 (numérique)

Découvrir la revue

\section{Citer cet article}

Chandioux, J. \& Guéraud, M.-F. (1981). MÉTÉO : un système à l'épreuve du temps. Meta, 26(1), 18-22. https://doi.org/10.7202/002213ar d'utilisation que vous pouvez consulter en ligne.

https://apropos.erudit.org/fr/usagers/politique-dutilisation/ 


\title{
Météo : un système à l'épreuve du temps
}

\author{
John Chandioux et \\ Marie-France Guéraud
}

\section{INTRODUCTION}

Au cours des dernières années, de nombreux communiqués de presse ou articles de revues spécialisées ont annoncé la mise en marché d'un système de traduction automatique révolutionnaire par une entreprise privée, la mise au point finale d'un système prototype par une université, ou encore, la mise en chantier d'un ambitieux système par un organisme public.

Dans le numéro de juin 1976 de la revue $M E T A$, on trouve un article intitulé «MÉTÉO, un système opérationnel pour la traduction automatique des bulletins météorologiques» décrivant un système prototype conçu et réalisé par le groupe TAUM de l'Université de Montréal.

En fait, le système n'est entré en exploitation que près d'un an plus tard, mais tourne, à la satisfaction du client, des destinataires et des traducteurs, depuis maintenant plus de trois ans.

\section{DU PROTOTYPE À L'EXPLOITATION}

Il est vraisemblable que le fait de confier le développement, la mise en exploitation et la mise à jour du système à une équipe déchargée de toute responsabilité de recherche, en l'occurrence une entreprise privée, a largement contribué au succès de l'entreprise, le secteur privé étant beaucoup mieux outillé pour faire face à ce genre de problème.

Les premiers essais ont été riches en surprises : le pourcentage de succès était inférieur à $50 \%$, au lieu des $80 \%$ escomptés; le système s'est avéré anormalement lent, et les logiciels n'étaient pas d'une fiabilité suffisante pour que l'exploitation puisse se faire sans risques.

Le passage de la version prototype à la version opérationnelle a nécessité les travaux suivants, qui se sont étalés sur près de dix-huit mois :

- Documentation, optimisation et développement des grammaires. Les grammaires actuelles sont nettement plus rapides, bien que près de deux fois plus grosses.

- Documentation, optimisation et modification des Système-Q, afin qu'ils répondent aux normes minimales de programmation en vigueur dans l'industrie. 
- Documentation et modification de l'environnement informatique au sein duquel les grammaires en Systèmes-Q sont exécutées.

\section{3. ÉVOLUTION DU MODÈlE LINGUISTIQUE}

Le modèle linguistique initial a été à la fois optimisé et augmenté au cours des trois dernières années; voici un aperçu des principales améliorations :

\section{Dictionnaire}

- L'entrée au dictionnaire des noms de région car les traducteurs se sont rendu compte, à l'usage, que la reconnaissance automatique des noms propres en contexte entraînait des erreurs de traduction lorsque certains mots étaient mal orthographiés ou inconnus du système :

\section{? RAIN IN THE EVENIN $\rightarrow$ PLUIE EN EVENIN}

Analyse

- L'ajout des transformations du type :

SHOWERS OR SNOWSHOWERS $\rightarrow$ SHOWERS OF RAIN OR SNOW THUNDERSTORMS LIKELY $\rightarrow$ POSSIBILITY OF THUNDERSTORMS AFTERNOON SHOWERS TOMORROW $\rightarrow$ SHOWERS TOMORROW IN THE AFTERNOON

- Un meilleur traitement des adverbes grâce à une sous-catégorisation selon leur comportement syntaxique.

— Le traitement de la complémentation des verbes. À l'origine, le système ne pouvait faire la distinction qu'entre les verbes transitifs et intransitifs. En fait, la traduction du verbe dépend de la présence ou de l'absence de sujet :

CLOUDY CLEARING OVERNIGHT $\rightarrow$ NUAGEUX SE DÉGAGEANT DURANT LA NUIT

CLEARING OVERNIGHT $\rightarrow$ DÉGAGEMENT DURANT LA NUIT

de la classe sémantique du sujet :

RAIN DEVELOPING... $\rightarrow$ PLUIE COMMENÇANT...

FOG DEVELOPING... $\rightarrow$ BROUILLARD SE FORMANT...

WIND DEVELOPING... $\rightarrow$ VENT SE LEVANT...

de la structure syntaxique de l'objet :

$\ldots$...BECOMING CLOUDY $\rightarrow$...DEVENANT NUAGEUX

...BECOMING FLURRIES $\rightarrow$...SE CHANGEANT EN AVERSES DE NEIGE

de la présence ou de l'absence de circonstanciel de temps:

CLOUDING OVER IN THE MORNING $\rightarrow$ SE COUVRANT DANS LA MATINÉE

CLOUDING OVER $\rightarrow$ SE COUVRANT DANS LA JOURNÉE 
pour ne citer que quelques-uns des problèmes qu'il est désormais possible de traiter.

\section{Transfert}

— L'insertion d'éléments sous-entendus dans le texte anglais:

\section{CLEAR AND COOLER TUESDAY $\rightarrow$ TEMPS CLAIR ET PLUS FRAIS MARDI}

- La redéfinition de la structure normalisée au profit d'une structure beaucoup plus aplatie, comme celle adoptée par le groupe du professeur Vauquois à Grenoble. L'expérience démontre qu'on y gagne en performance et que le développement du modèle linguistique peut se faire sans avoir à réécrire constamment la grammaire.

\section{Génération}

- La suppression du déplacement des circonstanciels de temps, qui introduisait des erreurs de traduction.

- La suppression de la génération morphologique du pluriel des noms au profit d'une entrée directe au dictionnaire. Ceci est d'autant plus logique qu'il n'y a pas d'analyse morphologique en anglais et permet, en outre, de rendre compte des noms au singulier dont la traduction est pluriel (CLOUD, FLURRY,...) ou inversement (MONDAYS,...).

\section{4. ÉVOLUTION DE L'ENVIRONNEMENT INFORMATIQUE}

L'environnement informatique des grammaires MÉTÉO a subi de grosses modifications depuis l'implantation initiale, et ceci d'autant plus que l'ordinateur ne tourne plus sous le même système d'exploitation. Nous ne citerons que celles qui ne nécessitent aucune connaissance en informatique :

- Le chargement des grammaires de traduction automatique se fait désormais toutes les cinq minutes, au lieu de toutes les dix minutes comme auparavant.

- Les codes de provenance des messages télégraphiques ne sont plus traduits par le programme de mise en forme des bulletins météorologiques, mais directement par les grammaires, ce qui facilite la mise à jour des listes de codes et simplifie considérablement le programme correspondant.

- La syntaxe des commandes de l'éditeur de texte utilisé par les traducteurs pour la révision a été modifiée en tenant compte de la position des touches du clavier. De plus, l'éditeur a été rendu moins «bavard», car les traducteurs étaient agacés par tous ses commentaires, surtout qu'ils ralentissaient le travail de révision, la vitesse maximale d'affichage à l'écran étant d'une demi-ligne par seconde.

- Le système a été doté d'une banque de données des communications traduites, afin de pouvoir réexpédier, à la demande du client, une communication qui n'est pas parvenue à destination. 
- Le Centre météorologique canadien est équipé de deux ordinateurs et il sera bientôt possible de faire tourner la traduction automatique sur le plus petit des deux, en cas de panne ou de mise hors service de l'autre.

\section{LE SYSTÈME MÉTÉO VU PAR LE TRADUCTEUR}

Une fois le texte traité par la machine et disponible pour l'édition, l'attention du traducteur est attirée par un affichage sur l'écran. Il aurait été possible de l'aviser par une sonnerie, mais cette solution a été jugée trop stressante et déshumanisante.

Le programme indique le nom du bulletin et offre le choix de le traiter ou de le refuser si c'est une répétition ou que des conditions d'urgence exigent son rejet. Les unités non traduites par la machine sont ensuite affichées à l'écran. Le traducteur peut, au besoin, obtenir comme contexte les unités précédente et suivante. Quand le bulletin est complet, l'expédition se fait elle aussi automatiquement.

Le temps moyen consacré à un bulletin ainsi traité est de 3,8 minutes, alors que la traduction entièrement manuelle exigerait de 30 à 40 minutes. $\mathrm{Ce}$ gain de temps permet donc à la même équipe de traiter davantage de texte sans augmentation de la charge de travail.

Les premières expériences de familiarisation avec ce système se sont déroulées il y a environ quatre ans. Tous les problèmes d'adaptation sont donc maintenant surmontés.

Il y eut d'abord l'appréhension devant du matériel nouveau, d'autant plus normale que la plupart des traducteurs n'avaient pas de formation technique ou scientifique. D'aucuns craignaient que de frapper la mauvaise touche ne bloque le programme ou, peut-être, ne fasse sauter la machine. Certaines erreurs suscitaient de plus de la part de l'ordinateur des réponses terrifiantes qui parlaient de «FATAL ERROR».

Ces craintes se sont évidemment dissipées avec le temps, et les traducteurs ont repris confiance, ce qui les a conduits à attendre en tout temps un rendement impeccable, tant du logiciel que du matériel. Évidemment, on s'habitue très bien à laisser le système faire $80 \%$ du travail en un temps record et, les jours de panne, il devient fort difficile de reprendre le collier de la traduction entièrement manuelle.

Un équilibre a maintenant été atteint entre les deux notions: machinemenace et machine-panacée. Les pannes sont acceptées avec davantage de sérénité et les changements de routine suscitent moins de heurts.

L'assurance vient avec l'habitude, et les traducteurs ont depuis reçu deux autres terminaux, consacrés à la traduction des textes qui ne sont pas soumis au système. Ils passent sans problème d'un logiciel à un autre, changent de type de clavier et d'écran sans même s'en rendre compte, et poussent presque les hauts cris quand il leur faut travailler sur cet engin préhistorique qu'est la machine à écrire électrique. 
6. L'AVENIR DU SYSTÈME MÉTÉo

À plusieurs reprises au cours des dernières années, on a envisagé la possibilité d'implanter le système MÉTÉO sur un ordinateur dédié. En effet, le coût d'exploitation actuel du système est relativement élevé (de l'ordre de cent mille dollars de temps de calcul par an) et l'installation du Centre météorologique canadien n'est pas orientée vers le traitement en temps partagé, ce qui alourdit le système. De plus, le niveau de saturation du système est tel, en particulier au niveau des entrées/sorties, qu'une communication n'est disponible pour révision qu'en moyenne dix-sept minutes après son arrivée, alors que la traduction ne demande que trente secondes.

Le principal obstacle à une implantation sur mini-ordinateur était sans doute l'absence de logiciel de traduction automatique qui puisse tourner sur ce genre de machine. Un métalangage facilement transportable a été développé et implanté sur micro-ordinateur. La société MICOM utilise déjà ce métalangage pour le développement de ses systèmes de détection des fautes d'orthographe en anglais et en français.

Le Secrétariat d'État examine actuellement la possibilité de faire de la traduction automatique sur micro-ordinateur et, plus précisément, de remplacer le système actuel par un système dédié.

Quoi qu'il en soit, c'est une affaire à suivre. 